\title{
Scripta
}

Revista Internacional de Literatura i Cultura Medieval i Moderna

\section{Da poeta a poeta, da traduttore a traduttore: il carteggio tra Umberto Saba e Tomàs Garcés}

\author{
From poet to poet, from translator to translator: \\ correspondance between Umberto Saba and Tomàs Garcés
}

\author{
GABRIELLA GAVAGNIN \\ gavagnincapoggiani@ub.edu
}

Universitat de Barcelona

Riassunto: Il rapporto personale tra Umberto Saba e Tomàs Garcés cominciò agli inizi degli anni Trenta e si interruppe poco prima della guerra civile spagnola. Quest'articolo analizza ed edita per la prima volta tutte le lettere scritte da Saba al poeta catalano che sono state conservate presso l'archivio della famiglia Garcés.

Parole Chiave: poesia, traduzione, Umberto Saba, Tomàs Garcés

Abstract: The personal relationship between Umberto Saba and Tomàs Garcés began in the early thirties and stopped just before the Spanish Civil War. This article analyses and edits for the first time all the letters written by Saba to the Catalan poet that have been preserved in the archives of family Garcés.

Keyword: Poetry, Translation, Umberto Saba, Tomàs Garcés 
Gabriella Gavagnin. Da poeta a poeta, da traduttore a traduttore: il carteggio tra Umberto Saba e Tomàs Garcés

Sulla relativa prontezza con cui Garcés segnala, commenta e traduce la poesia di Umberto Saba dei primi decenni del Novecento al lettore catalano abbiamo già richiamato più volte l'attenzione (Gavagnin 2001: 25-26 e 2005). Un giovanissimo Garcés scopre la lirica del poeta triestino tra le pieghe di un libro che gli capita tra le mani quando la sua conoscenza dell'italiano era ancora vacillante: l'antologia Poeti d'oggi con cui Papini e Pancrazi nel 1920 intendono fornire, in tempo reale, una mappatura della produzione poetica italiana. In quella messe di nomi già noti e di giovani promesse, Garcés discerneva le note di un poeta nuovo nell'orizzonte internazionale, ${ }^{1}$ verso il quale avvertí subito una particolare attrazione, poi confermata qualche anno dopo dalla lettura di libri come Preludio e fughe, Ammonizione ed altre poesie o Parole, che gli permisero di addentrarsi nell'opera di Saba. Lo affascinava soprattutto, come poi scriverà, la sobrietà dei simboli, la semplicità lapidaria dei versi, versi che considerava al tempo stesso austeri e pregnanti; ne ammirava poi quel felice abbinamento tra l'esperienza intima e vitale di cui si nutrivano, grazie a un implacabile e profondo scavo interiore, e il processo graduale di decantazione e di astrazione linguistica e stilistica capace di collocare tali sentimenti personali in una sfera di vaporosità e sospensione. Questo è quanto si ricava dalle diverse recensioni che ebbe modo di pubblicare sui periodici dell'epoca (Garcés 1928, 1933 e 1935).

La poesia di Saba non rappresentò solo una fortuita e fortunata lettura degli anni di formazione di un curioso giovane poeta. Dopo una ben più intensa, articolata e varia frequentazione della letteratura italiana, di ieri e di oggi, affiancata dall'esercizio sempre più spesseggiato e affinato di critico e di traduttore, nel corso degli anni trenta e alla vigilia di eventi storici che travolgeranno una vita culturale ricca di fermenti, innovazioni e inquietudini sperimentali su scala internazionale, Tomàs Garcés stabilisce un breve ma non sterile rapporto epistolare con Umberto Saba, iniziato appunto nelle prime settimane dell'anno 1933 e interrottosi nella primavera del 1936. In Garcés questo contatto con un uomo, un amico, che non riuscì mai a conoscere personalmente, che gli scriveva da una città che non ebbe mai occasione di visitare, si carica di una forte connotazione elegiaca, e conserva un che di misterioso e indeterminato che i trascorsi del tempo non cancellano. Negli appunti che Garcés scrive, ormai ottuagenario, in vista della seconda edizione della sua antologia Cinc poetes italians, ripercorre con accorata memoria quello scambio di missive. Quegli appunti, rimasti inediti e frammentari perché per motivi di salute la nuova presentazione non venne mai portata a termine (Manent 1984: 7), sottolineano soprattutto il vincolo stretto tra il poeta e la sua città, tra il poeta e il paesaggio evocatore di un sentimento poetico:

Escriure «Trieste» i recordar-me d’Umberto Saba, és tot u. Saba no el vaig conèixer mai. [...] Ens escrivírem, però. [...] A Trieste no hi he estat mai. En un angle dels sobres de les cartes de Saba, hi havia imprès uns mots: «Libreria Antiquaria» i en lletres més grans el nom del poeta-

\footnotetext{
1 La fortuna critica di Saba conosce in Italia i primi chiari riconoscimenti solo nella seconda metà degli anni venti. Garcés traduce per la prima volta Saba proprio a seguito della lettura dell'antologia di Papini e Pancrazi, pubblicando su La Revista nel 1921 «La noia», versione di una poesia tratta da Coi miei occhi. Per la stessa rivista tradusse poi, nel 1935, una poesia della raccolta Parole («Cendres»). A questa stessa raccolta attingerà in seguito per quattro delle cinque versioni sabiane incluse nella sua antologia Cinc poetes italians (Garcés: 1961).
}

SCRIPTA, Revista internacional de literatura i cultura medieval i moderna, núm. 5 / juny 2015 / pp. 253 - 264 ISSN: $2340-4841 \cdot$ doi:10.7203/SCRIPTA.5.6388 
llibreter (Això em recordava Salvat-Papasseit). I dintre d'una rodona, un dibuix enigmàtic amb una torre, un triangle, i algunes ratlles que semblaven, esquemàticament, la sortida del sol en l'horitzó marí. Via S. Nicolò 30, deia l'adreça. Era una gran avinguda, o un call del barri jueu? I Trieste, com devia ser? Saba, en una llambregada, ens ha mostrat en els seus versos, més d'un cop, la ciutat: la mira, dalt d'un turó estant: «Giunto alla vetta, scorsi in un fulgore / Trieste con le chiese e la marina». ${ }^{2}$

Nel gennaio del 1953 Garcés fa un breve viaggio in Italia per motivi di lavoro. In libreria trova, e acquista, l'edizione del Canzoniere di Saba del 1948. Di ritorno a Barcellona, scrive a Roma al suo vecchio amico Ettore De Zuani, il quale era stato negli anni venti direttore dell'Istituto Dante Alighieri di Barcellona e aveva propiziato il primo contatto epistolare tra Saba e Garcés. ${ }^{3}$ Gli chiede, così, se può fornirgli il recapito attuale del poeta triestino, perché è interessato a leggere «cosas más recientes que no tengo» ${ }^{4}$ di cui ha raccolto vaghe notizie in Italia, e anche perché vorrebbe spedirgli il suo ultimo libro di poesie, El caçador. Vorrebbe, insomma, riannodare un'antica amicizia proprio nello stesso modo in cui era a suo tempo sorta, attraverso uno scambio di letture poetiche. Ma il desiderio non poté realizzarsi.

Di quel contatto di prima della guerra resta la testimonianza diretta di una parte del carteggio. Tomàs Garcés serbava, infatti, otto missive ricevute dal poeta triestino, che corrispondono a due diversi momenti: le prime tre lettere sono ricevute tra gennaio e marzo del 1933, le altre, tra giugno del 1935 e marzo del 1936.

Il 20 gennaio 1933 Garcés scrive a Saba per chiedergli un esemplare di Ammonizione e altre poesie allo scopo di recensire il volume per La Veu de Catalunya. La risposta di Saba non si fa attendere: il 24 gennaio gli assicura che provvederà immediatamente all'invio del libro ${ }^{5}$ e gli raccomanda di

2 Tutti i manoscritti di Tomàs Garcés e la corrispondenza da lui ricevuta citati in quest'articolo sono conservati presso l'archivio familiare. Ringrazio per la disponibilità e generosità Carme Garcés, che mi ha permesso di consultarli.

3 È lo stesso Garcés, negli appunti inediti già citati, ad alludere alla mediazione di De Zuani, ricordandolo in modo impreciso come direttore dell'Istituto Italiano. In realtà, Ettore De Zuani non fu mai direttore dell'Istituto Italiano di Cultura di Barcellona, anzi, al momento della creazione dell'ente, nel 1934, aveva già da tempo lasciato la sede di Barcellona. Ettore De Zuani fu, invece, dal 1925 al 1929, direttore, oltre che professore, dell'Istituto Dante Alighieri, creato nel 1923 per unificare la scuola elementare, la scuola media e la serale (Santagati 2007: 325-329). Tuttavia, negli anni trenta tornò più volte in visita a Barcellona; una di queste soste, avvenuta durante un viaggio di ritorno in Italia dal Cile dove lavorava come addetto culturale all'ambasciata italiana (ibidem), risale appunto all'epoca del primo contatto epistolare tra Saba e Garcés.

4 Garcés conservava la brutta copia della sua lettera a Ettore De Zuani. Quanto alle pubblicazioni più recenti, nel dopoguerra Saba aveva pubblicato, in versi, Mediterranee e Uccelli-Quasi un racconto, e, in prosa, Scorciatoie e raccontini e Storia e cronistoria del Canzoniere.

5 Nella biblioteca di Tomàs Garcés si conserva l'esemplare che Saba gli spedì, numerato a mano e con dedica autografa dell'autore. Di Saba Garcés custodiva anche la raccolta Parole, pure ricevuta in dono dall'autore, il volume del Canzoniere acquistato in Italia (nell'edizione del 1948), e il libro di prose Ricordi Racconti (1910-1947) apparso nel 1956. Ringrazio Jordi Garcés per avermi reso possibile la consultazione del fondo di libri italiani della biblioteca di suo padre. 
Gabriella Gavagnin. Da poeta a poeta, da traduttore a traduttore: il carteggio tra Umberto Saba e Tomàs Garcés

fargli avere a sua volta l'eventuale notizia a stampa. ${ }^{6}$ Garcés ricambia l'omaggio spedendogli la sua ultima raccolta di poesie, Paradís. La risposta di Saba (27 febbraio) è molto vivace ed emozionata: vi si riflette la sorpresa e la curiosità che il libro gli ha suscitato, la sensazione gradita di scorgere intuitivamente una certa affinità in quel modo di far poesia, ma anche lo sconcerto di trovarsi di fronte a una lingua che gli risulta misteriosa e difficile da interpretare. Malgrado il carattere formale e incipiente del loro rapporto, gli chiede se può mettere a sua disposizione una traduzione di servizio di qualche poesia di quel libro. La preghiera non viene lì per lì esaudita, perché Garcés, poco tempo dopo, gli fa recapitare solo la recensione apparsa su La Veu de Catalunya. Sicché, allestiti in modo sbrigativo i dovuti ringraziamenti, Saba ritorna con grande e insolita insistenza sullo stesso punto, sulla stessa preghiera, posta con un senso di urgenza e impellenza, generata dal desiderio sincero di poter fruire della scrittura di un poeta la cui lingua è per lui poco accessibile. Anzi, lo è quanto basta per stimolare il suo interesse ma non tanto quanto gli occorre per trovare una risposta a tali sollecitazioni: che gli traduca anche solo una o due delle sue poesie, magari anche solo in prosa, ma che gli permetta di «capire più in là», di «capire del tutto», di addentrarsi insomma nel suo mondo poetico.

All'incirca due anni dopo, la richiesta è in parte appagata, in modo inaspettato e un po' eterodosso. Garcés, infatti, che prende l'iniziativa di riprendere il carteggio prima regalando a Saba un esemplare della sua nuova raccolta poetica, El senyal, e poi proponendogli di collaborare al progetto ancora in nuce dei Quaderns de Poesia, riesce a soddisfare in certo qualche modo il desiderio a suo tempo espresso da Saba. Così Garcés ricostruirà la vicenda a posteriori negli appunti inediti già citati:
En les tres primeres cartes, totes del 33, insisteix en el seu prec de les traduccions literals. No vaig trobar manera de complaure'l. [...] No fou fins a l'estiu del 1935, quan jo ja havia publicat «El Senyal», i ell en tenia un exemplar, que vaig aconseguir la traducció literal a l'italià de tres poemes d'aquell llibre. La va fer, un nen de l'Escola italiana. Tenia nou anys i n'era un destacat alumne. Aquell nen, fill d'un enyorat amic meu, es deia Manuel Duran i Gili, i és avui poeta i crític ben conegut i catedràtic a la Universitat de Yale. Estranyesa de Saba: només un poeta podia tenir la idea de fer-se traduir per un infant.

Effettivamente, Saba appare più stupito che appagato. Potrebbe sembrare quasi irritante l'insistenza con cui, anche così, Saba gli ripete per ennesima volta (e con un'enfasi evidenziata dall'uso delle maiuscole) che vorrebbe qualche altra traduzione, più canonica però, realizzata da un adulto, magari dallo stesso autore. In realtà, da quanto si deduce dalle lettere di Saba, ci fu una sequenza di due diverse spedizioni di manoscritti. Nella lettera del 24 agosto del 1935, infatti, si riferisce non a tre, ma a una singola poesia, ${ }^{7}$ aggiungendo che il fatto di doverla leggere nell'autografo del bambino-

6 Il fatto che Saba in questa prima missiva provveda a segnalare a Garcés il nuovo recapito di Ettore De Zuani conferma il ruolo di mediatore da questi svolto, perché sicuramente Garcés aveva menzionato il comune amico nella prima presa di contatto con Saba.

\footnotetext{
7 Poesia che, grazie all'immagine della luna che colpisce Saba («Bellissima poi la chiusa, con la luna del faro»), possiamo identificare con «Rossinyol sobre el port», i cui versi finali recitano appunto: «Per un instant, la lluna afluixa el seu timó / i neixen, mar endins, tornaveus inefables».
} 
Gabriella Gavagnin. Da poeta a poeta, da traduttore a traduttore: il carteggio tra Umberto Saba

e Tomàs Garcés

traduttore gli rende più ostico il compito di decifrarla. Nella lettera seguente, invece, si riferisce alle poesie ricevute (sicuramente le tre a cui allude Garcés nei suoi appunti), e non solo non protesta più per le difficoltà di comprensione grafica (segno che Garcés aveva provveduto a trascriverle prima di spedirgliele), ma ricambia la gentilezza con l'iniziativa di usare le versioni letterali e scolastiche diligentemente eseguite da Manuel Duran come ausilio per tradurre lui stesso quei testi. Sono quanto meno interessanti le osservazioni di Saba su questo suo esperimento di traduzione. Sottolinea, infatti, da un lato il carattere deliberatamente libero delle sue versioni poetiche, imprescindibile, spiega, per poter «salvare in forma italiana il tono particolare alla Sua poesia», per ricreare dunque il «tono», cioè gli aspetti stilistici e formali che connotano e personalizzano i versi originali. E per farlo, aggiunge, «ho dovuto ricorrere ad esclusioni e condensazioni», a una sorta di concentrazione, se non di soppressione, di elementi contenutistici. Quest'operazione avrebbe raggiunto, nel giudizio del traduttore, l'effetto di cancellare ogni carattere vicario del testo d'arrivo rispetto a quello di partenza, restituendo ai tre nuovi testi l'autonomia di testi ri-creati ( $\mathrm{Ne}$ sono uscite tre poesie che nessuno potrebbe dire tradotte»). Ma senza che l'artefice della ri-creazione se ne appropri stilisticamente, perché ne conserva la personalità che contraddistingueva le poesie nella lingua originale: «Un amico al quale le ho lette ieri a sera, negava infatti che fossero traduzioni, pur comprendendo subito che le poesie non erano mie. E sono infatti di un dolce poeta Catalano».

Purtroppo non conosciamo quelle versioni, tutte tratte come indica Garcés nei suoi appunti, dalla raccolta più recente, El senyal, ${ }^{8}$ perché, malgrado Saba si fosse impegnato per farle pubblicare sulle pagine della Gazzetta del Popolo di Torino, uno dei giornali più letti dell'epoca sul quale apparvero tra l'altro diverse poesie di Saba, le avverse circostanze politiche bloccarono l'iniziativa: in risposta alle sanzioni economiche applicate all'Italia dalla Società delle Nazioni per aver dato avvio alla guerra in Etiopia, il regime fascista proibí alla stampa italiana di pubblicare qualsiasi collaborazione giornalistica di autori appartenenti ai paesi sanzionisti. Come Saba faceva sapere a Garcés in quella che sarà l'ultima lettera da lui ricevuta, la redazione del giornale torinese aveva accettato volentieri di pubblicare quelle traduzioni e lo avrebbe fatto una volta terminate le sanzioni, a «orizzonte rischiarato», quando Saba si prometteva di riproporle allo stesso giornale in una versione leggermente perfezionata. Intanto, sul fronte opposto, la collaborazione di Saba ai Quaderns de Poesia sollecitata da Garcés approda a buon porto. Dopo aver lasciato cadere la prima idea di Saba di una ««Piccola Antologia» che comprendesse tre o quattro fra le mie poesie più significative (A mia moglie Tre vie - Il Borgo - Frutta - Erbaggi)», Garcés accoglie invece l'offerta di due componimenti, «Violino»e «Fontanella», che Saba gli presenta in qualità di quasi inediti dal momento che erano circolati solo sulle pagine della Gazzetta del Popolo. ${ }^{10}$

8 Sappiamo che una di esse è «Cavallets vora el mar», perché così lo ricorda Garcés nel prologo alla prima edizione di Cinc poetes italians. Supponiamo che un'altra delle tre sia la prima traduzione tradotta da Duran, cioè «Rossinyol sobre el port».

9 Sotto il titolo indicato da Saba, Due poesie, apparvero nel novembre 1935 nel quarto numero della rivista.

10 I due testi vi erano stati pubblicati da molto poco, il primo nel giugno e il secondo nel settembre di quello stesso

SCRIPTA, Revista internacional de literatura i cultura medieval i moderna, núm. 5 / juny 2015 / pp. 253 - 264

ISSN: 2340-4841 · doi:10.7203/SCRIPTA.5.6388 
Gabriella Gavagnin. Da poeta a poeta, da traduttore a traduttore: il carteggio tra Umberto Saba e Tomàs Garcés

Così si conclude, dunque, il doppio filo conduttore del carteggio. Al margine delle due vicende di ricezione e di traduzione reciproca, ${ }^{11}$ nelle lettere si toccano pochi altri temi, tra i quali conviene menzionare il ripetuto riferimento a altri scrittori e intellettuali rispetto ai quali Saba funge da intermediario. È lui stesso, per esempio, a segnalare (e a spedire) a Garcés l'opera prima di Pierantonio Quarantotti Gambini, suo amico oltre che per lungo tempo suo corrispondente epistolare. ${ }^{12} \grave{E}$ Garcés, invece, che gli chiede di fornirgli i recapiti di Corrado Govoni e di Giuseppe Ungaretti, forse per sollecitare loro collaborazioni per i Quaderns de Poesia. D'altro canto, i ringraziamenti e i saluti destinati «al Suo amico Signor R. d'Atos», ci portano a congetturare una qualche possibile partecipazione nel processo di traduzione dei versi di Garcés dello studioso italianista Ramon D’Alòs.

Trascriviamo in appendice, senza emendamenti, il testo integrale delle otto lettere conservate nell'archivio della famiglia di Tomàs Garcés. Sono tutte dattiloscritte su carta intestata alla Libreria Antiquaria e con firma autografa, fatta eccezione della lettera del 28 giugno 1935, scritta a mano su carta non intestata.

\section{Bibliografia}

Gavagnin, G. (2001) Poesia italiana i traduccions catalanes des de la Renaixença fins a la Guerra Civil, in De Leopardi a Ungaretti. Un segle de poesia italiana en versions catalanes de poetes-traductors, a cura de G. Gavagnin, Barcelona, Proa, pp. 9-30.

Gavagnin, G. (2005) L'italianisme de Tomàs Garcés del Renaixement als contemporanis, in Classicisme i Renaixement: una idea d'Italia durant el Noucentisme, Barcelona, Publicacions de l'Abadia de Montserrat, pp. 241-290.

Manent, M. (1984) «Nota preliminar a la segona edició», in Cinc poetes italians. Saba, Cardarelli, Ungaretti, Montale, Quasimodo, traducció de Tomàs Garcés, Barcelona, Empúries, pp. 7-8.

Garcés, T. (1928) «Umberto Saba «Preludio e fughe»», La Publicitat, 21-XII-1928.

Garcés, T. (1933) «Saba i la modernitat», La Veu de Catalunya, 11-III-1933.

Garcés, T. (1935) «Parole per Umberto Saba, R. Carabba, editore, 1935» [ma 1934], Quaderns de Poesia, 2, p. .

Garcés, T. (1961) Cinc poetes italians. Saba, Cardarelli, Ungaretti, Montale, Quasimodo, traducció i pròleg

anno, cfr. le note filologiche del Meridiano dedicato alla poesia di Saba (1994).

11 Stupisce che non ci sia alcun riferimento alla versione già citata della poesia tratta da Parole pubblicata da Garcés su La Revista nel 1935, mentre vi è puntualmente ricordata, nel post scriptum della lettera del 24 agosto 1935, la recensione al volume apparsa sui Quaderns de Poesia.

12 Il volume I nostri simili di Quarantotti Gambini che si conserva nella biblioteca di Garcés (Firenze, Edizioni di Solaria, 1932) è dedicato dall'autore al destinatario in data 20 marzo del 1933.

SCRIPTA, Revista internacional de literatura i cultura medieval i moderna, núm. 5 / juny 2015 / pp. 253 - 264 
Gabriella Gavagnin. Da poeta a poeta, da traduttore a traduttore: il carteggio tra Umberto Saba e Tomàs Garcés

de Tomàs Garcés, Barcelona, La Polígrafa.

Saba, U. (1994) Tutte le poesie, a cura di Arrigo Stara, introduzione di Mario Lavagetto, Milano, Mondadori.

Santagati, S. (2007) La Casa degli Italiani. Storia della comunità italiana di Barcellona (1865-1936), Barcelona, Editorial Mediterrània. 
Gabriella Gavagnin. Da poeta a poeta, da traduttore a traduttore: il carteggio tra Umberto Saba e Tomàs Garcés

\section{Appendice: Lettere di Umberto Saba a Tomàs Garcés}

Caro Signor Garcés. In evasione alla gradita Sua del 20 corr., oggi stesso Le spedisco — ben inteso in omaggio - una copia di «AMMONIZIONE ED ALTRE POESIE». Questo libro come rileverà dalla prefazione - comprende il testo e le scelte definitive delle mie poesie giovanili.

L'indirizzo attuale di de Zuani è presso la rivista L'ITALIA LETTERARIA, Roma, Salita San Nicolò da Tolentino.

Se parla di me in qualche rivista, abbia — La prego — la bontà di inviarmi quella parte di essa che mi riguarda.

Di ogni cosa con tutto il cuore La ringrazio, e La saluto affettuosamente, Suo riconoscente Saba

Caro Signor Garcés. Il suo libro mi ha messo alla tortura. Da quel pochissimo che ho potuto capire (intuire), mi sono immediatamente accorto che avevo da fare con un poeta indubitabile. Ma come fare, colla mia ignoranza del Catalano, a capire più in là? a capire del tutto almeno una sua poesia? Ho cercato fra tutti i miei amici se ci fosse qualcuno che conosce questa sua lingua, ma purtroppo invano. Vorrei (ma è chiedere troppo) che lei mi mandasse una traduzione, anche letterale, in prosa (italiana o francese) di qualcuna di queste poesie: per es. di quella — che è certo bellissima - che incomincia:

Oh, ciel, et voldràs reflectir

È impossibile che qualche sua poesia non sia stata tradotta in una di queste due lingue. Guardi se può accontentarmi.

Mi scriva. La saluto affettuosamente, suo

Saba 
Gabriella Gavagnin. Da poeta a poeta, da traduttore a traduttore: il carteggio tra Umberto Saba

\author{
e Tomàs Garcés
}

3

20 marzo 1933

Caro Signor Garcés. Ho ricevuto — inviato certamente da lei — il giornale di Barcellona che portava la sua recensione di «Ammonizione». Gliene sono infinitamente grato; ed ora attendo dalla sua amicizia che lei mi faccia avere una traduzione - italiana o francese - ANCHE IN PROSA, di qualcuna delle sue poesie contenute nel volumetto «Paradís». Non mi neghi, la prego, questo favore; io ho l'impressione netta che le sue poesie sono non solamente belle, ma anche che la loro bellezza è di un genere a me molto fraterno. Se lei può tradurmi, sia pure letteralmente, una o due di esse, io poi, confrontando la traduzione con l'originale, posso gustarle quasi come conoscessi l'originale.

Pierantonio Quarantotto Gambini, un giovane di ventidue anni, che ha scritta quella critica nell'Italia Letteraria, dalla quale lei ha riportate alcune frasi, è l'autore di un libro di racconti «I nostri simili», che ha avuto in Italia un successo insolito. Avendo letta la sua recensione, e vedendovi citato il suo articolo, mi ha incaricato di spedirle in omaggio un'esemplare (sic) del suo libro; cosa che ho fatta - oggi stesso - molto volentieri.

Le raccomando ancora una volta — se non sono indiscreto - la traduzione delle sue poesie, e la saluto affettuosamente,

suo devotissimo

Umberto Saba

Trieste; 28 giugno 1935

- Caro Signor Garcés. - La ringrazio per il dono del suo libro. Mi sono fatto tradurre alcune poesie, aiutandomi col testo, ed ho capito quel tanto che, insufficiente purtroppo per il mio diletto, è bastato tuttavia a confermare l'ammirazione che ho per Lei e l'intuizione che la sua poesia nasce da una vena miracolosamente schietta.

Vorrei ricambiare il dono del suo libro. Non so se l'editore (Carabba) Le ha spedito il mio ultimo libro «Parole». Nel caso non lo abbia e desideri riceverlo, mi scriva, che mi sarà cosa gratissima inviarglielo.

Con ammirazione ed affetto; suo Saba 
Gabriella Gavagnin. Da poeta a poeta, da traduttore a traduttore: il carteggio tra Umberto Saba e Tomàs Garcés

Caro poeta;

Come giustificarmi per il ritardo che ho messo a rispondere alla Sua ultima cara lettera? Dovrei raccontarLe - per farmi perdonare — tutte le noie che ho avute negli ultimi tempi. Perché la mia vita è molto difficile; e non la mia solamente...

Solo un poeta poteva avere l'idea di far tradurre una propria poesia da un fanciullo di nove anni. È stato un pensiero gentile e profondo... tuttavia avrei preferita la versione (e la calligrafia) di un fanciullo di trent'anni. Perché, oltre a tutto, vi sono, in quella versione, parole che non riesco a decifrare. Mi sono però fatta ugualmente un'idea abbastanza chiara della poesia. È quale la immaginava: uno squisito paesaggio che si riflette nell'anima, o che è esso stesso un riflesso d'animo. È come un'essenza del suo paese; ed ha al tempo stesso risonanze universali. Senza né stranezze, né oscurità, né immagini sforzate. Bellissima poi la chiusa, con la luna del faro. Quanto mi piacerebbe - non conoscendo il catalano - avere una traduzione dattiloscritta, anche solo letterale, di un gruppo di sue poesie!

Ringrazi il fanciullo Emanuele Duran per la sua fatica, la quale mi ha permesso, comunque, di intuire qualcosa della Sua arte, così lontana e certamente così vicina.

Non ho purtroppo nessuna poesia inedita per i Suoi Quaderni di Poesia. Non vuole qualcosa di già pubblicato, ma che certamente riuscirebbe nuovo ai Suoi lettori? Al caso, potremo metterci d'accordo; magari per una «Piccola Antologia» che comprendesse tre o quattro fra le mie poesie più significative (A mia moglie - Tre vie - Il Borgo - Frutta - Erbaggi). Oppure, se preferisce, una sola e breve.

TRADUCA LEI STESSO, COME PUÒ, QUALCUNA DELLE SUE POESIE E ME LE MANDI. Ci farò qualche correzione (per quanto riguarda la lingua) e poi le metterò a Sua disposizione.

Affettuosi saluti dal Suo amico Saba

P.S. Grazie anche per l'articolo su Parole. 
Gabriella Gavagnin. Da poeta a poeta, da traduttore a traduttore: il carteggio tra Umberto Saba

e Tomàs Garcés

6

Trieste, 26 ottobre 1935

Caro amico. La ringrazio per le sue poesie. E Le unisco quello che, in un momento felice, ne ho cavato.

È possibile però che lei rimanga male. Sono tutto quello che Lei può immaginare, meno che una traduzione letterale. Ma solo in questo modo ho potuto salvare in forma italiana il tono particolare alla Sua poesia. Per ottenerlo, ho dovuto ricorrere ad esclusioni e condensazioni. $\mathrm{Ne}$ sono uscite tre poesie che nessuno potrebbe dire tradotte. Un amico al quale le ho lette ieri a sera, negava infatti che fossero traduzioni, pur comprendendo subito che le poesie non erano mie. $\mathrm{E}$ sono infatti di un dolce poeta Catalano.

Adesso mi dica Lei che cosa ne dobbiamo fare. Vuole che le stampiamo in qualche giornale o rivista? Penso al Quotidiano «La Gazzetta del Popolo», che è il solo giornale che pubblichi, di quando in quando, qualche lirica scelta.

Nello stesso giornale ho pubblicato, tempo fa, due mie poesie, che pure le accludo. Se crede, può stamparle nella Sua Rivista, alla sola condizione di farmene avere a tempo le bozze di stampa. Per tutti i Suoi lettori - meno che per qualche italiano - sarebbero come se fossero del tutto inedite. Faccia però, senza complimenti, come crede meglio. Tengo così poco ad essere pubblicato...

Mi risponda subito per quanto riguarda le Sue poesie.

Affettuosi saluti dal Suo lontano amico Saba

Caro amico. Le rimando le bozze, con infiniti ringraziamenti. Il solo titolo generale che ho potuto dare è stato quello di «Due poesie».

Ho mandate le sue poesie alla «Gazzetta del Popolo» che è infinitamente più letta di qualunque rivista letteraria. Come mi pare di averLe detto, la Gazzetta non ha mai stampate traduzioni; il fatto però che non mi hanno rispedito il dattiloscritto, parrebbe significare che le stamperanno. Nel caso che questo non fosse, Le manderò ad una Rivista letteraria.

In un breve viaggio che ho fatto, ho lette le Sue poesie ad alcuni amici, alle quali esse sono piaciute immensamente. Ringrazi e saluti il Suo amico, il Signor R. D’Atos (sic), e a Lei i più affettuosi saluti dal suo Saba 
Gabriella Gavagnin. Da poeta a poeta, da traduttore a traduttore: il carteggio tra Umberto Saba

e Tomàs Garcés

Caro amico; Ha ragione di lamentarsi di me. Di quello che è - momentaneamente successo delle Sue poesie non ho colpa; ma la ho certamente di non averLe scritto. Dunque: ho mandate le tre poesie da me liberamente tradotte alla GAZZETTA DEL POPOLO di Torino; il gerente della quale $\mathrm{mi}$ ha risposto che le avrebbe stampate molto volentieri, ma che, fino che durano le sanzioni, non può pubblicare componimenti di scrittori appartenenti ai paesi sanzionisti. A orizzonte rischiarato, il Giornale sarà lieto di stamparle. Intanto, io non ho più il dattiloscritto, e Le sarei immensamente riconoscente se mi mandasse una copia di quello che, a suo tempo, ho inviato a Lei. Appena la cosa sarà fattibile, lo rimanderò, probabilmente con qualche "perfezionamento» allo stesso Giornale, il quale è molto diffuso ed è il solo dove, essendo stampato, un poeta possa avere la speranza di essere letto.

Mi creda che sono molto dispiacente del ritardo. Non Le ho scritto solo perché mi dispiaceva darLe questa notizia.

Non sono in relazione con Govoni. A Ungaretti scriverò oggi stesso, esprimendogli il Suo desiderio.

Mi mandi il dattiloscritto, La prego

Suo, con ammirazione ed affetto Umberto Saba 